\title{
Usefulness of the European Epidemic Intelligence Information System in the management of an outbreak of listeriosis, Belgium, 2011
}

M Yde ${ }^{1}$, M Naranjo ${ }^{1}$, W Mattheus ${ }^{1}$, P Stragier ${ }^{1}$, B Pochet ${ }^{2}$, K Beulens ${ }^{2}$, K De Schrijver ${ }^{3}$, D Van den Branden ${ }^{3}$, V Laisnez ${ }^{3}$, W Flipse $^{3}$,

A Leclercq ${ }^{4}$, M Lecuit ${ }^{4}$, K Dierick' ${ }^{1}$, S Bertrand (Sophie.Bertrand@wiv-isp.be) ${ }^{1}$

1. Communicable and Infectious Diseases, Scientific Institute of Public Health, Brussels, Belgium

2. Federal Agency for the Safety of the Food Chain, Belgium

3. Infectious Disease Control Unit, Department of Public Health Surveillance, Flemish Agency for Care and Health, Antwerp, Belgium

4. French National Reference Centre and WHOCC for Listeria, Institut Pasteur, Paris, France

Citation style for this article:

Yde M, Naranjo M, Mattheus W, Stragier P, Pochet B, Beulens K, De Schrijver K, Van den Branden D, Laisnez V, Flipse W, Leclercq A, Lecuit M, Dierick K, Bertrand $\mathrm{S}$. Usefulness of the European Epidemic Intelligence Information System in the management of an outbreak of listeriosis, Belgium, 2011. Euro Surveill.

2012;17(38):pii=20279. Available online: http://www.eurosurveillance.org/ViewArticle.aspx?Articleld=20279

A cluster of time-linked cases and the identification of a clonal strain suggest the occurrence of an outbreak of listeriosis in Belgium in 2011, presumably due to the consumption of hard cheese made with pasteurised milk and produced by a Belgium manufacturer. The outbreak clone was identified as Listeria monocytogenes serovar $1 / 2 \mathrm{a}$, sensitive to arsenic and cadmium and of multilocus sequence typing MLST-type 37. Food investigation of this outbreak was facilitated by the European Epidemic Intelligence Information System and data exchanged between French and Belgium listeriosis surveillance systems.

\section{Introduction}

Listeria monocytogenes, a Gram-positive bacterium, is a ubiquitous organism in the environment and a facultative intracellular food-borne pathogen. Infections occur through ingestion of contaminated food. The bacterium causes listeriosis, which is characterised by bacteraemia or meningitis. Infection during pregnancy can lead to abortion. The incubation time of listeriosis is estimated from two to 88 days. Immunocompromised patients, the elderly, pregnant women and neonates are particularly at risk of developing symptomatic disease [1]. Due to its high mortality rate (approximately $25 \%$ of the patients) and hospitalisation rate (approximately $97 \%$ of the patients), timely and accurate isolate characterisation is essential to identify outbreaks [2].

The Belgian National Reference Centre for Listeria (BNRCL) receives annually between 40 and 70 strains of human clinical cases $(53,64$ and 43 cases in 2008, 2009 and 2010, respectively), representing an annual incidence of three to six cases/million habitants. The vast majority of them are sporadic cases, infected with unrelated molecular strains. Contrary to the Flemish speaking community of Belgium, listeriosis is mandatorily notifiable in the French speaking community.
In the present study we report an outbreak of 12 cases of human listeriosis in Belgium in 2011. The strain linked to the outbreak was characterised by serotyping, metal resistance typing, pulsed field gel electrophoresis (PFGE) and multilocus sequence typing (MLST) $[3,4]$. The microbiological characteristics were then communicated within the European Union (EU) via the Epidemic Intelligence Information System (EPIS), a platform tool allowing national surveillance systems to exchange information regarding current or emerging public health threats with a potential impact in the EU. This allowed the identification of a hard cheese as a likely source of the outbreak.

\section{Methods}

\section{Case definition}

For this outbreak, a person was considered a case if the $L$. monocytogenes strain isolated from this person had the same Apal/Ascl PFGE pattern as the outbreak strain as well as the serotype (1/2a) and MLST-type 37 .

\section{Outbreak investigation}

Cases diagnosed with listeriosis received a standardised questionnaire aimed at detecting risk factors for Listeria and at collecting information about their food history in the two month before the onset of symptoms. The patients gave verbal consent to answer the questionnaire.

Listeria monocytogenes bacteria were isolated from blood and ascites fluid of patients by the clinical laboratories. The isolates were sent to the BNRCL for confirmation of the identification and typing.

Strain identification was carried out with API Listeria microgallery (bioMérieux, France). Serotyping was conducted according to a standard protocol using a commercial agglutination test (Denka Seiken, Tokyo, Japan) 
for somatic (O) and flagellar $(\mathrm{H})$ antigens [5]. Strain susceptibility to arsenic and cadmium was determined according to McLauchlin et al. [3]. Antibiotic susceptibility was tested by E-test (bioMérieux); the following antibiotics were studied (Susceptible/Resistant (S/R) breakpoints in $\mu \mathrm{g} / \mathrm{ml}$ ): ampicillin (2/4), amoxicillin $(4 / 16)$, gentamicin $(4 / 16)$, vancomycin $(4 / 32)$, erythromycin (0.5/8), tetracycline (4/16), ciprofloxacin (1/4), chloramphenicol (8/32) and trimethoprim/sulphamethoxazole (2/4). Molecular typing was performed on all human isolates. Pulsed-field gel electrophoresis (PFGE) was done following the United States (US) PulseNet protocol after deoxyribonucleic acid (DNA) digestion with the Apal and Ascl enzymes [6]. Band pattern analysis was performed with ImageMaster video documentation system (Amersham Pharmacia Biotech) and BioNumerics (Applied Math). Multilocus sequence typing was performed according to the method described by Ragon et al. [4], which is based on allelic analysis of seven housekeeping genes and enables the comparison of the obtained sequences to an online accessible database [7].

\section{FIGURE 1}

Pulsed-field gel electrophoresis AscI and ApaI profiles of the outbreak of human listeriosis clone, Belgium, 2011
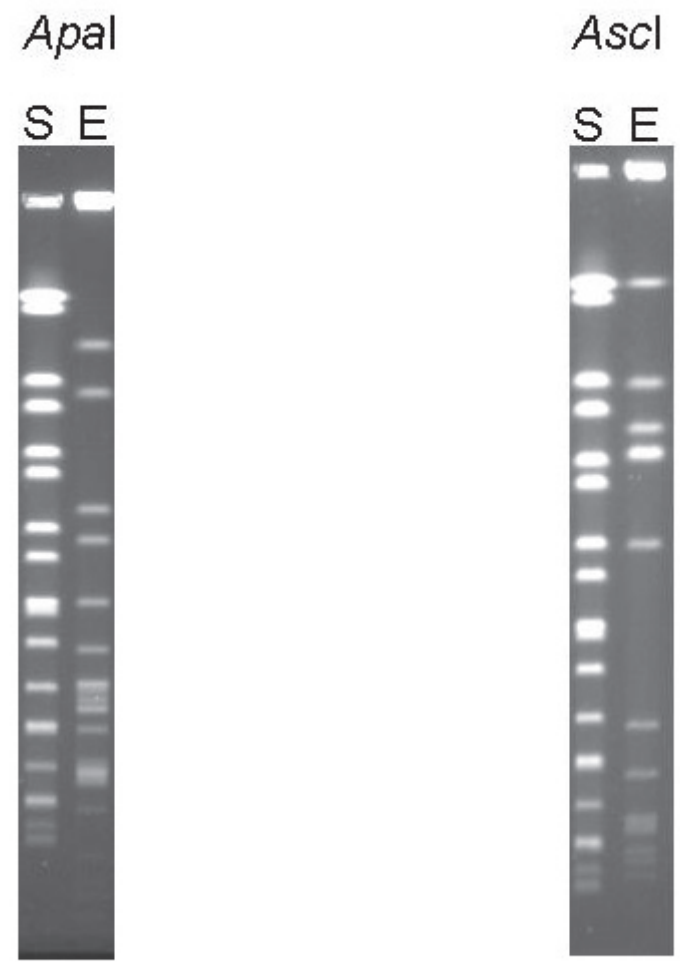

S: Salmonella Braenderup as reference, E: Listeria monocytogenes outbreak clone.
International enquiry

In order to find out if the outbreak strain had occurred in clinical samples or food isolates, in particular within the EU, the microbiological characteristics of the outbreak strain were communicated via EPIS, a communication platform established by the European Centre for Disease Prevention and Control (ECDC).

\section{Results}

\section{Outbreak investigation}

Between 4 February and 1 March, 2011 BNRCL received three clinical isolates of $L$. monocytogenes from respective patients hospitalised at a same hospital in Antwerp. The responsible strain was confirmed at BNRCL as $L$. monocytogenes serovar $1 / 2 \mathrm{a}$ and sensitive to arsenic and cadmium (SS). Molecular typing of the three clinical isolates showed a clonal origin based on their similar combined PFGE Ascl/Apal profile (Figure 1) and MLST-type 37.

An investigation for possible nosocomial infection was immediately started at the hospital and was inconclusive. Meanwhile, BNRCL continued to receive clinical isolates, with the same microbiological and molecular characteristics from other hospitals in Belgium confirming the detection of an outbreak. A total of 12 isolates positive for the outbreak strain were identified as of June 2011, from 12 hospitalised patients, three of whom were known to have been initially hospitalised for other illnesses than listeriosis. Eleven of the isolates were derived from blood and one from ascites fluid. All were sensitive to the nine antibiotics tested.

The outbreak began in February when two respective patient isolates with the outbreak strain were received by BNRCL, it reached a maximum in March and April, with four respective positive isolates, and declined in May and June with one positive isolate respectively (Figure 2).

The patients' characteristics are presented in the Table. The overall mean age was 77.3 years (range: 56-86 years). Five patients were male and seven female. The mean age of the male patients was 82 years and the mean age of the female patients was 74.6 years. No pregnancy-related case was reported. An underlying disease was reported for eight patients while this was unknown for the others. During the period of the outbreak four patients died. For two of the deaths, the sepsis caused by the Listeria outbreak strain was a significant contributing factor, while for the remaining two the direct cause was underlying disease.

Six patients lived in the province of Antwerp; the remaining cases lived in five different provinces distributed almost nationwide (Figure 3 ).

In search of the source of infection, the BNRCL database was consulted. A hard cheese isolate was 


\section{FIGURE 2}

Cases of listeriosis outbreak by month of diagnosis in the Belgian National Reference Centre for Listeria, Belgium, $2011(n=12)$

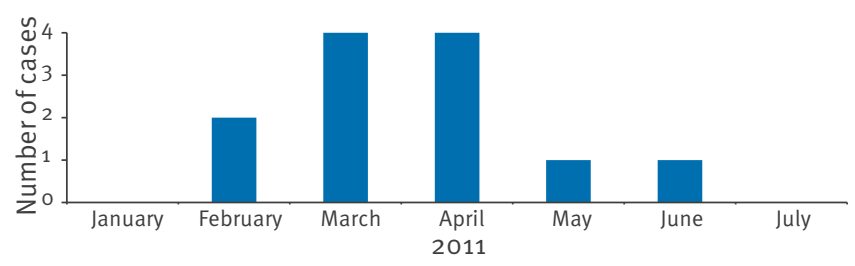

identified with similar microbiological characteristics as the outbreak clone. This food isolate was received at the BNRCL a year before, in conjunction with the monitoring programme of the Belgian Federal Agency for the Safety of the Food Chain.

\section{International enquiry}

The clinical strains typing results were submitted to EPIS of the Food and Waterborne Diseases and Zoonoses Programme of the ECDC, to investigate for the previous isolation of human/food strains with the same microbiological characteristics particularly in other EU Member States. The inquiry (Reference 2011-04-05-497) was created on 05 April 2011 and 13 countries replied. Twelve EU and European Free Trade Association (EFTA) countries and the US reported no similar unusual increases of listeriosis. At the same time of the Belgian outbreak, the French National Reference Centre for Listeria reported an indistinguishable food strain isolated subsequent to a food alert in the Département du Nord, an area, bordering Belgium. Food isolates with the same strain as the outbreak strain all originated from hard cheese made from pasteurised milk (called Pavé $d u$ Nord). The cheese was manufactured in Belgium and imported to France where it was sliced, packaged and sold in supermarkets. Enumeration of L. monocytogenes in this sliced and packaged hard cheese was low, around $20 \mathrm{cfu} / \mathrm{g}$. In addition, two hard cheeses raw material samples had been analysed and found to meet the microbiolical safety criterium of less than $100 \mathrm{cfu} / \mathrm{g}$ of L. monocytogenes [8]. The Belgian cheese manufacturer and its distributor nevertheless decided to recall different batches of hard cheese from the national and international market, and issued a warning to the public. Four alert notifications were launched by the Rapid Alert System for Food and Feed (RASFF) of the European Commission, on the presence of L. monocytogenes in hard cheese: Three in Belgium (Alert Notification 2011.0374; 2011.0511; 2011.0619) and one in France (Alert Notification 2011.132).

At the Belgian cheese manufacture plant, an inspection was launched to identify the origin of contamination. The Federal Agency for the Safety of the Food Chain ordered the operator to send all isolated L. monocytogenes strains to the BNRCL for further typing. The $B N R C L$ received two cheese isolates and three cheese surface swab strains with the same microbiological characteristic of the outbreak clone. The samples had been taken at the manufacture plant. Several cheese samples taken at one hospital with human cases proved negative for the presence of $L$. monocytogenes.

The strains isolated from the patients and from hard cheese were indistinguishable but it remained to be established if the patients had consumed the suspected food. To this aim, all patients (or their physicians) received a questionnaire, yet only four of the 12 patients responded. No information about the patients with a fatal outcome could be collected. No responders remembered having eaten hard cheese from the suspected manufacturer, yet the delay between disease and questionnaire filling was long (ranging from one to four months). Nevertheless, after the food inspection of the cheese manufacture plant and appropriate sanitary measures taken on site, no more cases with the outbreak clones was recorded at BNRCL.

\section{Discussion}

Centralised surveillance of the human Listeria cases is essential for the early detection of outbreaks and for the organisation of an immediate and appropriate response [9]. The investigation and control of foodborne outbreaks involve different actors and requires data centralisation from different sectors such as clinical medicine, epidemiology, food safety, risk communication and management. In this study, the outbreak

\section{TABLE}

Characteristics of outbreak cases of listeriosis, Belgium, $2011(n=12)$

\begin{tabular}{|c|c|c|c|}
\hline $\begin{array}{l}\text { Case } \\
\text { number }\end{array}$ & $\begin{array}{l}\text { Isolation site } \\
\text { of the strain }\end{array}$ & $\begin{array}{l}\text { Clinical } \\
\text { presentation }\end{array}$ & Comorbidity \\
\hline 1 & Blood & NA & NA \\
\hline 2 & Ascites fluid & Peritonitis & NA \\
\hline 3 & Blood & NA & $\begin{array}{l}\text { Respiratory } \\
\text { insufficiency }\end{array}$ \\
\hline 4 & Blood & $\begin{array}{l}\text { Septicaemia } \\
\text { and peritonitis }\end{array}$ & Liver insufficiency \\
\hline 5 & Blood & Septicaemia & Multiple myeloma \\
\hline 6 & Blood & Septicaemia & Bronchial carcinoma \\
\hline 7 & Blood & Septicaemia & $\begin{array}{l}\text { Chronic obstructive } \\
\text { pulmonary disease }\end{array}$ \\
\hline 8 & Blood & NA & NA \\
\hline 9 & Blood & NA & NA \\
\hline 10 & Blood & Septicaemia & NA \\
\hline 11 & Blood & Septicaemia & $\begin{array}{l}\text { Liver cirrhosis and } \\
\text { type } 2 \text { diabetes }\end{array}$ \\
\hline 12 & Blood & NA & NA \\
\hline
\end{tabular}

NA: Not available. 


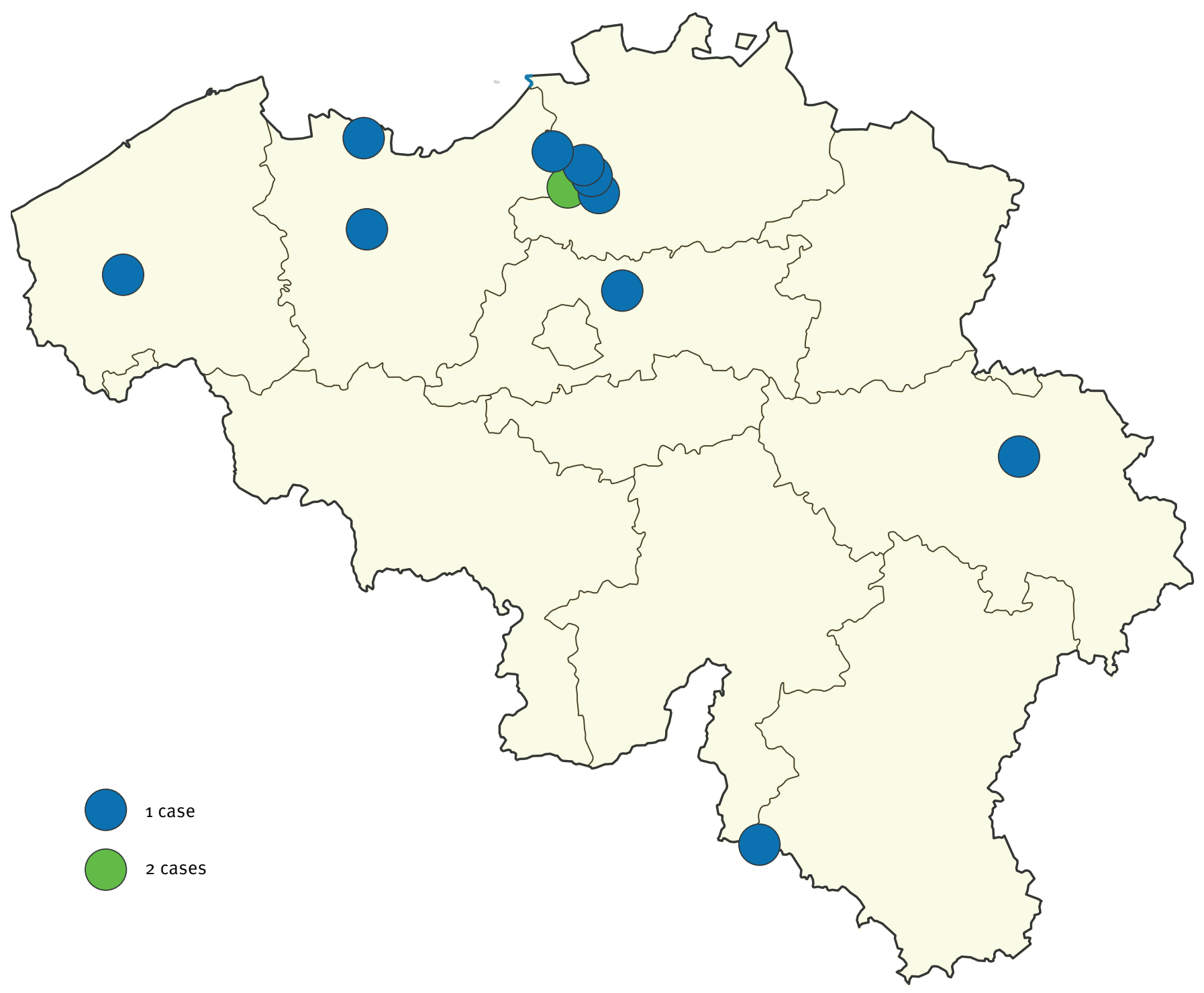

was detected because the BNRCL received strains from clinical laboratories and performed subtyping of all the strains. Although Belgian clinical laboratories transfer their clinical isolates to the BNRCL favouring BNRCL to play a central role in the early detection of human clusters at the community and national level, this is done on a voluntary basis. It is therefore estimated that the BNRCL receives between 70 to $80 \%$ of the total number of isolated clinical strains, offering potential for an underestimation of the number of cases in this outbreak.

By means of this centralised data collection, additional information regarding trends, emerging agents, transmission routes, groups at risk and specific risk factors can be obtained in order to manage an outbreak and to eliminate the source of contamination.
Nevertheless, a challenge for outbreak management is to trace back the food source of infection. The long incubation time of listeriosis makes it difficult to successfully interview patients and identify the source of infection. The Cantaloupe outbreak in the US underlined also the difficulty to detect quickly foods which are not classically listed as a source of contamination in humans and at the origin of outbreaks [10]. Moreover, food investigation in outbreak management is difficult when an imported contaminated product is at the origin of national cases. In this study, food investigation was facilitated via EPIS and an alert was created on 5 April 2011, to which 13 countries replied. The same day information from France pointed to the Belgian cheese manufacturer, and this prompted the withdrawal of batches of the suspected cheese from the market. As the Netherlands also imported cheese from that manufacturer, contacts between the Dutch investigators 
and the BNRCL were facilitated by the ECDC Food and Waterborne Diseases and Zoonoses network. No cases linked to this outbreak were reported by the Netherlands.

Within the epidemiological investigation about this outbreak, use of the food questionnaire proved to be unsuccessful, probably as it was not conducted soon enough after the diagnosis of listeriosis: only four patients responded and no one remembered having eaten hard cheese from the suspected cheese manufacture plant. This is quite understandable, also given the relatively old age of the patients, the long incubation time of the disease and the lapse of time between the onset of the illness and receiving the written questionnaire. Furthermore, unpackaged sliced hard cheese is not labelled at the retail level, making it more difficult for the consumer to link the information about the manufacturer with the cheese. To limit these types of problems it is important to question the patients verbally, immediately after the diagnosis of listeriosis. Unfortunately the Belgian questionnaire did not inquire patients on the consumption of hard cheese from pasteurised milk, although hard cheese from unpasteurised milk is included; hard cheese from pasteurised milk should be added to the questionnaire in the future.

In the food investigation part of this outbreak, there are only microbiological indications that hard cheese was the source of contamination. Complete DNA sequence of food and patient outbreak strains could be more conclusive. Results of the internal investigation of the cheese manufacturer suggested that the rind of the hard cheese was contaminated with L. monocytogenes originating from a machine that rotates the cheese during riping, which was shown to be contaminated. Meanwhile, measures were taken to disinfect this rotator. This observation underlined the fact that, as for example slicers in the ready-to eat meat industry, equipment may play an important role in L. monocytogenes contamination of a product [11].

It is very exceptional to assign hard cheese as the causative food of listeriosis cases, unlike soft cheese which is recognised as an at risk product [11]. Microbiological criteria for $L$. monocytogenes in hard cheese ripened or not, are established for healthy people ( $100 \mathrm{cfu} / \mathrm{g}$ ) but, for hospitalised people, should be modified to absence. Even low levels of $L$. monocytogenes in hard cheese pose a risk to immunocompromised persons and this type of cheese is often served in hospitals or in elderly homes. In the United Kingdom, it was therefore recommended that food served to hospital patients be free from L. monocytogenes $[12,13]$. In our view as a result of this study, a clarification of European regulation on microbiological criteria for food served in hospital should be investigated to protect this particular type of consumer.
We believe that our results underline the need for mandatory notification of listeriosis in Member States in order to facilitate food and human outbreak investigations. In addition, our investigation shows the importance of continuous exchange of human and food data between EU Member States based on an established network, combined with molecular typing surveillance of listeriosis in Europe, based on harmonised and recognised methods for early detection and management of national or European outbreaks. Due to the international marketing of foods or raw food materials and the free movement of people between countries, we consider that the surveillance of listeriosis only at national level is currently insufficient to protect the consumer.

\section{References}

1. Rocourt J, Bille J. Foodborne listeriosis. World Health Stat Q. 1997;50(1-2):67-73.

2. Barbuddhe SB, Chakraborty T. Listeria as an enteroinvasive gastrointestinal pathogen. Curr Top Microbiol Immunol. 2009;337:173-95.

3. McLauchlin J, Hampton MD, Shah S, Threlfall EJ, Wieneke AA, Curtis GD. Subtyping of Listeria monocytogenes on the basis of plasmid profiles and arsenic and cadmium susceptibility. J Appl Microbiol. 1997;83(3): 381-8.

4. Ragon M, Wirth T, Hollandt F, Lavenir R, Lecuit M, Le Monnier A, et al. A new perspective on Listeria monocytogenes evolution. PLoS Pathog 2008;4(9):e1000146.

5. Seeliger HP. Modern taxonomy of the Listeria group relationship to its pathogenicity. Clin Invest Med. 1984;7(4):217-21.

6. Martin P, Jacquet C, Goulet V, Vaillant V, De Valk H; Participants in the PulseNet Europe Feasibility Study. Pulsed-field gel electrophoresis of Listeria monocytogenes strains: the PulseNet Europe Feasibility Study. Foodborne Pathog Dis. 2006;3(3):303-8.

7. Listeria monocytogenes MLST database. Paris: Institut Pasteur. [Accessed 20 Sep 2012]. Available from: http://www.pasteur. $\mathrm{fr} /$ recherche/genopole/PF8/mlst/Lmono.html

8. European Commission. Commission Regulation (EC) No $2073 / 2005$ of 15 November 2005 on microbiological criteria for foodstuffs. Official Journal of the European Union. Luxembourg: Publications Office of the European Union. 22.12.2005:L 338. Available from: http://eur-lex.europa.eu/ LexUriServ/LexUriServ.do?uri=0J:L:2005:338:0001:0026:EN: PDF

9. Denny J, McLauchlin J. Human Listeria monocytogenes infections in Europe--an opportunity for improved European surveillance. Euro Surveill. 2008;13(13): pii=8082. Available from: http://www.eurosurveillance.org/ViewArticle. aspx?Articleld $=8082$

10. Centers for Disease Control and Prevention. Multistate outbreak of listeriosis associated with Jensen Farms cantaloupe--United States, August-September 2011. MMWR Morb Mortal Wkly Rep. 2011;60(39):1357-8.

11. Oliver SP, Jayarao BM, Almeida RA. Foodborne pathogens in milk and the dairy farm environment: food safety and public health implications. Foodborne Pathog Dis. 2005;2(2):115-29.

12. Coetzee N, Laza-Stanca V, Orendi JM, Harvey S, Elviss NC, Grant KA. A cluster of Listeria monocytogenes infections in hospitalised adults, Midlands, England, February 2011. Euro Surveill. 2011;16(20): pii=19869. Available from: http://www. eurosurveillance.org/ViewArticle.aspx?Articleld=19869

13. Fretz R, Pichler J, Sagel U, Much P, Ruppitsch W, Pietzka AT, et al. Update: Multinational listeriosis outbreak due to 'Quargel', a sour milk curd cheese, caused by two different L. monocytogenes serotype 1/2a strains, 2009-2010. Euro Surveill. 2010;15(16): pii=19543. Available from: http://www. eurosurveillance.org/ViewArticle.aspx?Articleld=19543 